\title{
A Ride around the Blockchain
}

\section{WILL BLOCKCHAIN TAKE FINANCIAL PROCESSES TO A BETTER PLACE?}

By Sherree DeCovny

The term "blockchain" tends to be used loosely because, technically, it is the technology that supports the Bitcoin cryptocurrency. When financial industry participants talk about blockchain, however, they often are referring to blockchain-like distributed ledger systems that can be used for various applications.

In April 2016, CFA Insti-

Blockchain is more about

enhancing business

processes than about

replacing existing

Proof-of-concept efforts are under way, but wide adoption is a long-term play.

More security, resiliency, and transparency along with reduced costs could boost investor confidence and trust, but industry tute published the "Fintech Survey Report," which sheds light on the topic of automation in financial advice. Respondents picked blockchain as the technology that will have the second-greatest impact (after robo-advisers) on the financial services industry five years from now. The top three areas survey respondents cited as potentially being affected by blockchain technology are clearing and settlement, technology. buy-in is critical to success. alternative currencies, and commercial banking. Other areas include fund administration, asset servicing, capital market infrastructure, other banking functions, asset management, and real estate. Interestingly, about one-third of the 775 survey respondents were unable to comment, indicating a low level of awareness of what blockchain technology is and how it might affect them. (For more information on the fintech survey, see "Understanding the Fintech Hype" by Rhodri Preece on page 52 of this issue.)

Indeed, distributed ledger systems are being tested in payment processing, trade finance, and back-office recordkeeping functions, especially interorganizational ones such as clearing and settlement. They are being considered for trading OTC derivatives, swaps, bonds, commodities, and unregistered securities, as well as syndicated loans, repos, and warehouse receipts. Real estate landed at the bottom of the list of impact areas in the survey, although distributed ledger systems are already being implemented for land registry.

Blockchain is not a technology replacement but more of a business process enhancement in the context of financial services, according to William Mougayar, an investor and advisory board member at the Ethereum Foundation and author of two books on this subject.

"At the end of the day, it's about $80 \%$ business and 20\% technology," he says. "It's about changing the way the processes are currently being implemented."
Thousands of developers worldwide have been working on the Ethereum blockchain platform for the past couple years. The core blockchain code has been written, and programmers are currently writing what are known as "smart contracts." Smart contracts are written in extremely precise programming languages, such as Solidity, so when users read the code they can see precisely what it is going to do. Because competent Java, LIS, or C++ programmers can teach themselves Solidity in a matter of weeks, a shortage of skills does not appear to be a major issue.

"The technology aspect of blockchain is a bit overblown," agrees Sviatoslav Rosov, CFA, analyst for capital markets policy at CFA Institute. "It's not a particularly complicated piece of software for computer programmers."

The point of blockchain technology is to move the industry toward a shared recordkeeping ecosystem. Banks and other organizations could accomplish that using legacy technology, but a lack of cooperation has prevented them from doing so. However, blockchain is catalyzing the industry to at least consider collaboratively developing new standardsbased, interoperable systems; abandoning legacy technology; and enabling new business models.

\section{ADOPTION LIFE CYCLE}

Blockchain will likely be implemented in three phases. The first phase is proof of concept, which is now being done. The second phase is early adoption. The third phase is wider adoption and implementation of protocols and standards that are accepted by everyone.

Several organizations are doing proof-of-concept projects in trading, clearing, and settlement. Overstock.com issued a \$25 million debt offering using the Bitcoin blockchain in August 2015 as a proof of concept. The SEC approved Overstock's plans to issue shares on its t0 platform that will resemble cryptocurrency (although the shares will not be Bitcoin), and transactions will be conducted using blockchain technology.

NASDAQ is working on a proof of concept in Estonia to enable proxy voting using blockchain technology. In addition, the NASDAQ Private Market has done a proof of concept to manage cap tables, issue equity, and arrange special terms and rights using its Linq product.

ICAP, a market operator and provider of post-trade risk mitigation and information services, successfully completed a proof of concept in its Post Trade Risk and Information (PTRI) division in February 2016. Using bilaterally executed spot/forward foreign exchange block trades, the PTRI division imported matched messages from Traiana's Harmony network and employed blockchain infrastructure to convert 
the messages in real time to blockchain-based smart contracts. These were in turn distributed to nine representative participant nodes on the blockchain network. From there, trades could be used to provide additional services, such as valuation, compression, and reporting.

Over the coming months, ICAP will discuss with participants how it can access the new golden data source and start work to realize technology savings in disparate systems. At the same time, it wants to ensure compliance with regulations intended to make markets safer and more efficient.

Moreover, R3, a consortium of more than 50 financial institutions, is collaborating on research, experimentation, design, and engineering to help advance blockchain to meet banking requirements for identity, privacy, security, scalability, interoperability, and integration with legacy systems. R3 recently unveiled Corda, its shared ledger platform specifically designed to record, manage, and synchronize financial agreements between regulated financial institutions. It is heavily inspired by and captures the benefits of blockchain systems without the design choices that make blockchain inappropriate for many banking scenarios.

Over the next three to five years, there will be some attempts at commercializing the technology. The Australian Stock Exchange (ASX) is already planning to put some back-office functions onto the blockchain and use it in its day-to-day business rather than as just a proof of concept done in a sandbox. If the exchange is successful, it may be motivated to implement blockchain technology for other functions. The ASX is well positioned to be an early adopter because the organization is vertically integrated.

Establishing standards for interoperability, participation and access, documentation, and regulation is a long-term play in the capital markets. SWIFT (a provider of secure financial messaging services) and Accenture (a management consulting services company) recently published a joint position paper titled "Delivering an Industry Standard Platform through Community Collaboration" investigating how distributed ledger technologies could be used in financial services. The study identified gaps between existing distributed ledger technology solutions and industry requirements, including eight critical factors that need to be addressed for the technology to achieve industry-wide adoption. These include strong governance, data controls, compliance with regulatory requirements, standardization, identity framework, cybersecurity and cyberdefense, reliability, and scalability.

\section{SECURITY, RESILIENCY, AND TRANSPARENCY}

Blockchains are distributed and decentralized by design, meaning that there is no single point of failure. If one part of the network goes down, the other parts keep humming. To this end, some believe it is an ideal environment for resiliency and security. Additionally, blockchain could provide a higher level of transparency and visibility to the total transaction life cycle and streamline all the processes between the order and the clearing and settlement of the transaction.

Theoretically, blockchain enables the transfer of securities and cash to occur simultaneously, which reduces risk.
But some stakeholders note that $\mathrm{T}+2$ is a market and regulatory convention and legacy technology is capable of settling transactions in $\mathrm{T}+0$. Besides, many trades are netted, so the amount that needs to clear and settle at the end of the day is a fraction of the total trades.

"Instantaneous settlement may not necessarily be the goal," says Mougayar. "Gross settlement of every trade would introduce more risk, whereas liquidity is better reflected with a netting that takes into account the time interval between trade and settlement. That said, for certain markets, a time reduction would be beneficial."

Rosov is skeptical about getting a critical mass of participants on board to enable real-time settlement via the blockchain.

"Blockchain is one way to get $\mathrm{T}+0$, but it is not the only solution or a silver bullet," he says. "You still need the cooperation and to get the market participants to agree to this new approach."

From his perspective, organizations have enough trouble upgrading common software applications such as Microsoft Windows and Office. Blockchain technology involves "upending everything, root and branch," so it would have to be significantly better than the status quo for firms to take on all the impediments. That being said, trading has changed rapidly with the adoption of disruptive new technologies, and a displacement of legacy systems could be accelerated if the benefits are clear.

Potential disintermediation is another issue. By providing a mechanism for automatically verifying the identity and status of assets, the peer-to-peer technology facilitates the movement of value, capital, and goods without going through an intermediary. That could have significant implications for financial institutions.

Startups are competing with the incumbent financial firms. These new companies can leapfrog legacy technologies and launch their services on blockchain. For example, UK-based Clearmatics is developing a decentralized clearing network, which will settle and clear transactions for OTC markets on a blockchain-based platform.

"The incumbents will be competing with these startups," says Mougayar. "If they don't improve and change, then the buy side will go to these new networks because they will be faster, cheaper, and more modern."

To prevent disintermediation, financial firms should analyze what parts of their business may be threatened by blockchain and be proactive in implementing blockchain themselves to create new services.

\section{CONFIDENCE, CRISIS, AND COSTS}

In February 2016, CFA Institute published "From Trust to Loyalty: A Global Survey of What Investors Want," which canvassed retail investors in 10 markets and institutional investors in 6 markets. The results showed that institutional investors want their investment firms to be held to a high ethical standard while also being transparent about fees and protecting their data. Increased fees and data/confidentiality breaches were the second and third reasons cited for leaving an investment firm. 
When institutional investors were asked how important certain attributes are, low fee levels ranked third on the list after proactive communications about market dynamics and credentialed employees. Half of the respondents cited "early adopter of new technology that benefits my portfolio" as the most important attribute.

Further, 29\% of institutional investors reported that they believe a financial crisis in the next three years is likely. That being said, 49\% responded that they are prepared to manage their portfolio in a crisis.

The security, resiliency, and transparency of blockchain could help to boost investor confidence and trust that was lost as a result of the recent financial crisis and technology glitches. Having a golden record of transactions would certainly facilitate a bankruptcy resolution should a "too big to fail" institution collapse.

If blockchain ultimately reduces transaction costs, the savings could be passed on to investors. But as Rosov points out, some robo-advisers charge $0.25 \%$. How much lower can fees go? The bigger issue is that investment firms are being asked to

justify their fees and performance to clients and the industry. If clients determine the performance does not justify the fees they are charged, then the level is irrelevant.

With blockchain technology, capital and collateral provisions are reduced because transactions are settled in real time, thus lowering credit risk. But Rosov questions whether credit risk is simply being traded for systemic risk by eliminating buffers and safety valves.

"Blockchain is not making any firms, investment projects, or cash flows less risky, as far as I'm aware," he says. "In the true fashion of the human race, we are confident that we are designing an ever-more-perfect system, and actually, we're just kicking the risk upstairs."

Finally, even if all exchanges KEEP GOING implemented blockchain, people would still complain about market structure, just as they always have.

"Not everyone in the market can

"Fintech Survey Report": www.cfainstitute.org/ Survey/fintech_survey.PDF

"From Trust to Loyalty: A Global Survey of What Investors Want": www.cfainstitute.org/ investortrust

"Chips Off the Old Blockchain," CFA Institute Magazine (November/December 2015) [www.cfapubs.org] win, and the ones who don't win tend to not like it," Rosov concludes.

Sherree DeCovny is a freelance journalist specializing in finance and technology.

\section{$\rightarrow$ FIERACAPITAL}

\section{WHERE PERFORMANCE MEETS PEACE OF MIND.}

MONTREAL • TORONTO • CALGARY • VANCOUVER • HALIFAX • NEW YORK • LOS ANGELES • BOSTON • SAN FRANCISCO

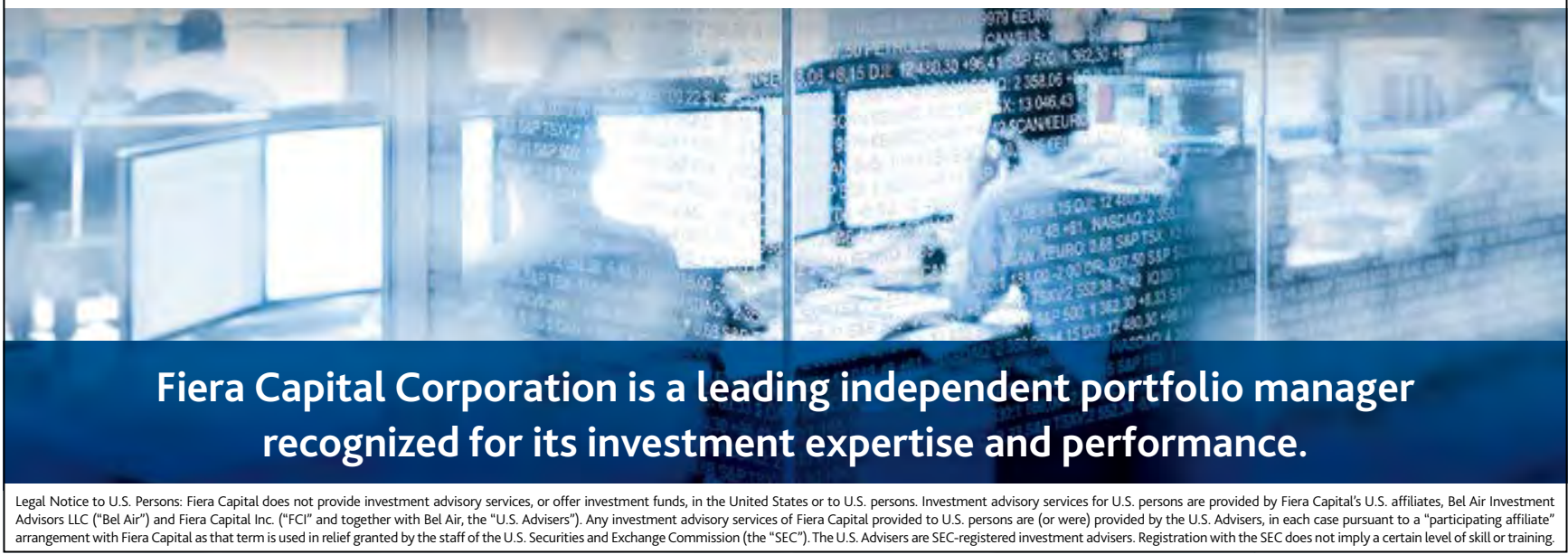

